\title{
LA GRÈVE DÉFAITE
}

Noëlle Burgi

\author{
Association Vacarme | «Vacarme »
}

2004/4 $n^{\circ} 29$ | pages 6 à 9

ISSN 1253-2479

ISBN 9782915547672

Article disponible en ligne à l'adresse :

http://www.cairn.info/revue-vacarme-2004-4-page-6.htm

\section{Pour citer cet article :}

Noëlle Burgi, « la grève défaite », Vacarme 2004/4 (n²9), p. 6-9. DOI 10.3917/vaca.029.0006

Distribution électronique Cairn.info pour Association Vacarme.

(C) Association Vacarme. Tous droits réservés pour tous pays.

La reproduction ou représentation de cet article, notamment par photocopie, n'est autorisée que dans les limites des conditions générales d'utilisation du site ou, le cas échéant, des conditions générales de la licence souscrite par votre établissement. Toute autre reproduction ou représentation, en tout ou partie, sous quelque forme et de quelque manière que ce soit, est interdite sauf accord préalable et écrit de l'éditeur, en dehors des cas prévus par la législation en vigueur en France. Il est précisé que son stockage dans une base de données est également interdit. 


\section{la grève défaite}

\author{
par Noëlle Burgi
}

$1^{\mathrm{er}}$ mai 1984-:

le gouvernement Thatcher annonce la fermeture de la mine de Cottonwood, dans le Yorkshire. La grève que les mineurs engageront quatre jours plus tard durera un an - mais la trouée ouverte par son échec dans les lignes du mouvement ouvrier et des organisations syndicales ne se refermera plus. À travers elle, le paradigme néo-libéral pourra s'engouffrer et s'étendre aux dimensions du monde. Retour sur une stratégie, et chronologie d'un désastre.

Noëlle Burgi a notamment publié :

L'État britannique contre les syndicats, Kimé, 1992. Fractures de l'État-nation (dir), Kimé, 1994.
$\mathrm{M}$ argaret Thatcher était encore dans l'opposition quand elle a commencé à se préparer à la grande grève des mineurs qui se déclencherait en mars 1984 et durerait exactement un an. La portée historique de cet événement ne saurait être sous-estimée-: agissant dans une situation stratégique complexe qu'elle a partiellement configurée et dont elle s'est habilement saisie, la Dame de fer a ainsi permis au néolibéralisme anglo-saxon d'imposer au monde entier ses normes et son idéologie.

La grève avait été minutieusement préparée. Au lendemain de son élection en 1975 à la tête du parti conservateur, Margaret Thatcher avait mis sur pied des groupes de travail chargés d'élaborer sa " contre-révolution politique et sociale » dont le succès passait par une épreuve de force monumentale avec le mouvement ouvrier. Un rapport confidentiel de l'époque, le rapport Ridley, définissait les houillères comme le " champ de bataille le plus probable " et préconisait une série de mesures préventives (accumulation de stocks de charbon, recrutement de camionneurs non syndiqués dans les transports routiers, etc.) pour briser la résistance des "ennemis de l'intérieur " avant même qu'ils ne s'organisent. Par ailleurs, d'autres mesures étaient envisagées pour affûter les armes de ceux qui serviraient le moment venu d'alliés indispensables au pouvoir politique. Ainsi, après la victoire électorale des tories en 1979, la police a développé une force nationale mobile, responsable devant un comité de coordination, dotée de matériels et d'entraînements spécifiques. Les capacités d'intervention pénale ont été renforcées à travers une première série de réformes limitant la légalité des conflits du travail, et engageant la responsabilité civile ou pénale des syndicats et des militants. Une nouvelle politique énergétique orientée prioritairement vers le nucléaire fut annoncée dès octobre 1979, initiative suivie par une augmentation des stocks de charbon et des importations de pétrole, une inversion du rapport charbon-pétrole dans la production d'électricité et la création d'une commission spéciale chargée de préparer des plans pour réprimer tout conflit du travail important.

Alors qu'il était techniquement prêt à affronter les mineurs, le gouvernement tory a cependant reculé devant une menace de grève en 1981. La tactique était habile. Margaret Thatcher ne perdait rien à différer provisoirement son plan de restructuration des charbonnages-: les moins malins croyaient à une faiblesse gouvernementale, les plus avertis comprenaient qu'il fallait encore " colorer l'eau [...], ce qui signifiait conditionner le climat de l'opinion publique ". Comme l'a expliqué un responsable politique de l'époque, "le problème était de savoir comment faire face au pouvoir mythique de la NUM [National Union of Miners, le syndicat des mineurs]. C'était clair en février 1981. Les éditoriaux d'alors, de droite ou de gauche, écrivaient tous-: "Terrible métier..., hommes décents..., donnez leur l'argent-!” Autrement dit, 
"nous n'étions pas prêts, mentalement. Les syndicats ont eu l'impression qu'on nous avait envoyés au tapis. C'était délibéré-: ils recevraient notre poing la prochaine fois-!" ” Sachant que la grève allait mettre en œuvre une violence inouie dans les rapports sociaux, cette temporisation en vue d'une préparation psychologique de la société comportait une double dimension, pragmatique et spectaculaire. D’un côté, il fallait s'assurer que la police n'hésite pas à faire la démonstration de sa force et de ses nouvelles capacités. Le moment venu, on lui demanderait en effet d'étaler au grand jour le pouvoir répressif de l'État, et notamment d'aider à transformer en délits des agissements qui ne l'étaient pas. Elle n'avait jamais joué un tel rôle national, n'y était pas prête en 1981 et, à en croire des témoignages exprimés pendant et après le conflit, a conçu une grande amertume à servir ce but.

De l'autre côté, Thatcher voulait que la défaite planifiée de la grève serve d'exemple pour tous, à commencer par les salariés et leurs représentants. Par là, elle voulait infléchir radicalement le rapport des forces économiques et sociales et rendre illégitime, sinon illégale, toute " pratique restrictive» de la part des syndicats, toute «-crispation » sur les acquis sociaux, et plus largement toute revendication de justice ou d'équité sociales. Elle avait bien choisi son terrain. Les mineurs, considérés comme le fer de lance de la classe ouvrière, avaient acquis une réputation d'invincibilité après leurs grèves de 1972 et 1974, qui renversèrent le gouvernement conservateur d'Edward Heath. En outre, la nature de leur travail rude et dangereux dans un monde souterrain hostile, ainsi que la culture du travail, sinon du dévouement à l'effort, transmis de génération en génération pour s'adapter et se réapproprier dignement l'univers de la mine, en avaient fait les héros de l'industrie, les héros du travail. Cette représentation était encore prégnante dans l'imaginaire collectif au début des années 1980, et elle faisait sens pour beaucoup de mineurs malgré l'évolution (inégale selon les bassins) de leurs conditions de vie et de travail depuis la Seconde guerre mondiale.

En 1983, Margaret Thatcher nommait à la tête des charbonnages britanniques Ian MacGregor, un manager issu du privé qui venait de faire ses preuves dans la sidérurgie en la restructurant en un temps record (1980-1983). Son message était clair-: elle était prête.

Les mineurs, eux, s'apprêtaient à affronter un gouvernement intransigeant, tout en sachant qu'ils n'obtiendraient ni de la confédération syndicale TUC (Trade Union Congress) ni du Labour un engagement suffisant dans la lutte. Depuis 1979, le mouvement ouvrier était sur la défensive. Le taux de syndicalisation avait fortement chuté, le taux de chômage avait grimpé jusqu'à 13-\% et le niveau de combativité, en baisse, était gravement affecté par l'échec de grands conflits (comme ceux de la sidérurgie en 1980, des employés de la santé et de ceux des chemins de fer en 1982). Il en est résulté de fortes divisions internes au TUC. Après la défaite électorale du parti travailliste en juin 1983, le TUC et le Labour ont accéléré leur recentrage " néoréaliste », reflétant leur désarroi face à l'offensive néolibérale.

Soutenu par la faible et très minoritaire gauche du parti travailliste, Arthur Scargill, le dirigeant charismatique de la NUM, n'ignorait pas qu'il lui serait très difficile de mobiliser l'ensemble des mineurs. Quoique les mineurs fussent, en apparence, inégalement menacés par la restructuration des charbonnages (certains bassins comme celui du Nottinghamshire étant bien lotis avec des puits à la pointe de la modernité), ils étaient cependant décidés à combattre les politiques du gouvernement et de la direction de l'entreprise publique. En témoigne la stricte observation par tous les mineurs d'une grève des heures supplémentaires qui a précédé pendant dix-neuf semaines le déclenchement de leur ultime grande grève. Mais ils étaient divisés sur la stratégie et la tactique à suivre. Après s'être, en 1981, déclarés favorables à 86-\% à l'action dans l'industrie, ils avaient été 61-\% à en rejeter le principe en octobre 1982 et en mars 1983. C'est que la revendication ne porterait plus sur les salaires ou les conditions de travail, mais sur la fermeture des puits " non économiques ", c'est-à-dire, in fine, sur l'emploi. Quelle contre-proposition opposer à ceux qui, en fermant les puits, fermaient aussi, a pu dire une femme, "la vie des gens »-?

Sincèrement convaincu qu'il était de son devoir de conduire ses troupes vers la transformation socialiste de la société, Arthur Scargill ne croyait pas aux bienfaits des luttes corporatistes. Mais, quelle que fût son idéologie, le piège s'était refermé sur lui. Ou il se rangeait à la position néoréaliste maintenant dominante parmi ses alliés potentiels, et il acceptait sans broncher le destin réservé aux mineurs, ou il décidait de se battre, mais il lui était difficile, sinon impossible, étant donné le rapport de forces et les visées de la «contre-révolution » conservatrice en marche, d'imaginer autre chose qu'une lutte rétablissant le statu quo ante. Il a choisi la deuxième solution et s'est lancé dans la bataille en affichant une intransigeance digne de celle de ses adversaires.

La grève fut déclenchée le 5 mars 1984 dans le Yorkshire, fief d'Arthur Scargill, quand fut annoncée, contrairement à ce qui avait été promis, la fermeture du puits de Cortonwood. À cette première provocation s'en ajouta aussitôt une deuxième. Le lendemain, le 6 mars, la direction des charbonnages rendit public un plan partiel de restructuration entraînant la suppression de 20000 emplois et la fermeture d'une vingtaine de puits en 198485 . Le 12 mars, Scargill proclama la grève nationale sans consulter le TUC ni sa base. 90 mines sur 176 se rallièrent au mouvement qui s'étendit à travers les bassins du pays et toucha une forte majorité des 184000 mineurs (ils seront majoritaires pendant onze mois sur douze). Dans 
les communautés minières, les femmes s'impliquaient en participant aux piquets de grève et aux manifestations de masse, en effectuant des tournées de ville en ville et dans les entreprises pour appeler à la solidarité. Parmi les dissidents, on nota surtout la région clé du Nottinghamshire. Jusqu'à l'été, tandis que les négociations entreprises échouaient (28 mai, 13 juin, 18 juillet), le rapport de forces semblait se dessiner en faveur des mineurs. Le gouvernement fut même surpris de leur capacité de résistance, mais n'entendait pas leur laisser l'avantage et prit directement parti dans le conflit, tant par le biais policier, judiciaire et politique, qu'en intervenant sur le cours des négociations. De ce fait, ces dernières aboutirent toutes à une impasse. Sur le terrain, les mineurs ne parvenaient pas à consolider le rapport de forces politique et social qui leur semblait initialement favorable. En juillet et septembre 1984, deux grèves nationales des dockers furent étouffées dans l'œuf, faute d'entente entre les dirigeants syndicaux-; une «Triple Alliance » entre cheminots, sidérurgistes et mineurs ne put être mise sur pied-; surtout, une menace de grève des porions et agents de sécurité, qui aurait entraîné la cessation d'activité de toutes les mines, fut annulée le 25 octobre grâce à un compromis avec la direction des charbonnages.

Forts de l'inaction des autres syndicats et de la direction travailliste qui ne cessait de dénoncer «la violence » dans la grève, Ian MacGregor et le gouvernement manœuvrèrent sur le champ de bataille en s'appuyant notamment sur un Comité national des mineurs au travail qu'ils avaient suscité à partir de la région non-gréviste du Nottinghamshire. Fin novembre 1984, les fonds de la NUM furent bloqués, en exécution d'une décision de la Haute Cour. Une campagne gouvernementale pour la reprise du travail lancée avant les fêtes de Noël commença à prendre sérieusement en janvier 1985. En février, un accord fut passé entre la direction des charbonnages et le TUC, prévoyant l'arrêt de la grève sans garanties sur les fermetures de puits. Cet accord fut rejeté par un congrès extraordinaire de la NUM-; mais, le 6 mars 1985, isolés, épuisés par un an de lutte, les mineurs et leurs représentants en viennent à décider d'eux-mêmes la reprise du travail sans avoir obtenu la moindre concession.

"Vous ne reverrez plus jamais une chose pareille », a pu dire à propos de la grève un représentant du syndicat des mineurs. Il avait raison. C'était bien le dernier très grand conflit dans le monde industriel, celui qui allait clore l'époque du keynésianisme, tourner la page de l'État-providence, accélérer le tournant vers la financiarisation de l'économie et introduire le néolibéralisme comme pratique sociale et comme principe idéologique dominants. Son importance historique tient à la simultanéité de la « révolution conservatrice » initiée aux États-Unis par Ronald Reagan (élu en 1980) et de la «-contre-révolution » thatchérienne qui, en remportant là une victoire décisive, ouvrait la voie à la globalisation du nouveau paradigme néolibéral.

La victoire était décisive car le succès de ce projet économique, politique et idéologique passait par un affaiblissement radical du monde du travail et de ses organisations représentatives. Au lendemain de la grève, plus aucun syndicat n'était en mesure de résister à la redéfinition des règles du jeu dans les relations professionnelles et sur le marché du travail. Par un mélange de déréglementations et de nouvelles réglementations, fut renforcé à l'extrême le principe de l'employeur seul juge-: seul juge de sa "capacité de payer ", de la viabilité de son entreprise, de l'organisation et des conditions de travail, de l'opportunité d'embaucher, de licencier, de négocier ou de consulter. Et puisque tous les acquis sociaux durement conquis depuis un siècle se trouvaient du même coup taxés d' "-archaïsmes ", chacun a été invité à endosser la responsabilité de sa situation sociale, désormais considérée comme une caractéristique individuelle, un trait marquant de l'être-: le chômage, la pauvreté laborieuse et même la privation d'avenir qui peut en résulter sont "gérés » par les institutions, expliqués à travers le prisme de l'employabilité individuelle. Avec, pour conséquence,

\section{Au lendemain de la grève, plus aucun syn- dicat ne pouvait résister à la redéfinition du jeu dans les relations profes- sionnelles.}

une moralisation de la vie sociale, productrice de règles répressives visant au redressement des esprits et des corps : si l'on est «éloigné de l'emploi ", c'est que l'on est trop « assisté »-; c'est que le travail n'est pas assez contraint (le workfare) et le contrôle social insuffisant.

Cette évolution n'indique pas simplement un renversement du rapport de forces entre capital et travail-: en reconstituant une "multiplicité de rapports de force immanents au domaine où ils s'exercent ", elle suggère une transformation profonde du pouvoir au sens où l'entendait Foucault. À ne s'en tenir qu'à la question du travail et de l'emploi, il est en effet remarquable que la pratique et l'idéologie néolibérales aient remodelé les représentations, les jugements, les attentes, les espoirs et les agissements des acteurs sociaux, et cela très majoritairement, bien au-delà et en deçà des personnes, des 
lieux et des instruments censés détenir, alimenter et institutionnaliser la domination économique et politique. La mise en cause de l'emploi, en ce qu'il confère à chacun un statut ou, pour le dire avec les mots d'Amartya Sen, en ce qu'il offre à chacun la possibilité de vivre une vie valorisante pour soi, a été un des leviers essentiels de cette transformation.

Il va de soi que les outils traditionnels du pouvoir politique ont été largement mobilisés pour modifier ainsi le devenir des sociétés. Comme en témoigne le rôle de la police et de la justice durant la grève des mineurs. Mais la méthode thatchérienne ne s'arrêtait pas là. Pour réussir ce que Ian MacGregor, désignant ce conflit, a appelé un grand " coup de poker ", pour en stabiliser les effets et poursuivre la mise en œuvre de son programme, la Dame de fer a concentré le pouvoir entre les mains d'un exécutif tout à sa dévotion. Non sans une contradiction apparente, puisque le discours dominant s'appliquait à convaincre de la nécessité de "faire reculer les frontières de l'État ».

La méthode s'étant perfectionnée et ayant essaimé depuis pour devenir un mode de "gouvernance" à prétention universelle, on perçoit mieux la logique reliant ces deux formes terminales, en réalité très complémentaires, d'exercice du pouvoir. Loin de se limiter aux privatisations, la «réduction de l'État» a désigné et désigne toujours l'abandon par celui-ci de ses fonctions d'arbitre et de garant de la justice sociale. Nonobstant le jugement que l'on portera sur l'accomplissement de ces fonctions depuis la Seconde guerre mondiale, cet abandon est une des sources principales du "déficit démocratique " qui surprend régulièrement, par exemple à l'occasion des échéances électorales, les détenteurs du pouvoir politique. Leur étonnement est peut-être authentique, car cette démission n'a jamais été assumée comme telle. Au contraire, si son efficacité a supposé que soit décentralisée, régionalisée, communautarisée, voire parcellisée l'exécution de décisions prises dans une grande opacité (et généralement hors des enceintes parlementaires), sa légitimation est passée par deux voies complémentaires. D'une part, l'adoption d'un discours et de méthodes inspirées des techniques managériales de gestion pour " moderniser » l'État et les institutions - en revêtant les choix politiques d'un idéal d'objectivité et de neutralité, cette première voie transformait l'art de gouverner en technique normalisatrice à prétention universelle. D'autre part, pour sauvegarder les apparences démocratiques, de multiples procédures consultatives, "participatives ", de «proximité » ou non, ont été inventées. Censées promouvoir, par exemple, le « dialogue social », elles avaient et ont toujours pour finalité d'engager la responsabilité d'un maximum de participants dans la mise en œuvre de décisions prises ailleurs. Vingt ans après la grève des mineurs britanniques, le néolibéralisme et sa complexe redistribution du pouvoir se sont infiltrés par tous les pores de la société. 